\title{
Uso de nuevos anticoagulantes orales (NACO) en la prevención del ictus y la embolia sistémica en pacientes con fibrilación auricular no valvular (FANV)
}

\author{
PAULA RodRÍGUEZ GÁLVEZ \\ al318135@uji.es \\ LAURA VALERO VERche \\ al316975@uji.es \\ SONIA PI RUANO \\ al321875@uji.es \\ AlBA SÁNCHEZ MARTíN \\ al316975@uji.es \\ LUIS LIZÁN TUDELA \\ lizan@uji.es
}

\section{Resumen}

Introducción: Las herramientas de ayuda para la toma de decisiones compartidas son materiales, con información fiable basada en estudios científicos, sobre una determinada enfermedad o problema de salud en cuestión. Las herramientas son utilizadas para ayudar a las personas a sentirse más informadas y cómodas, para tomar decisiones sobre un tema de salud como el expuesto en el trabajo, teniendo en cuenta sus preferencias y valores. Además, facilitan la relación de las personas afectadas por una enfermedad y los profesionales sanitarios con el objetivo de llegar a una toma de decisiones conjunta. Metodología: En este artículo se ha creado una herramienta para la toma de decisiones compartidas, realizándose un análisis y una comparación de los distintos anticoagulantes presentes en la actualidad para la prevención del ictus y la embolia sistémica en pacientes con fibrilación auricular no valvular (FANV). Resultados: La presente herramienta, que consta de un tríptico para poder ser utilizado en Atención Primaria, quiere ser probado dentro de las prácticas que realicemos el próximo curso y de esta manera comprobar su utilidad.

Palabras clave: FANV; anticoagulantes; toma de decisiones compartidas; herramienta; preferencias.

\section{Abstract}

Introduction: Shared decision-support tools are materials, with reliable scientificbased information, about a given disease or health problem. Tools are used to help people feel more informed and comfortable, Make decisions on a health issue such as the one exposed in the work, taking into account their preferences and values, and 
facilitate the relationship of people affected by a disease and health professionals with the goal of reaching joint decision-making. Methodology: This article has created a tool for shared decision making, with an analysis and comparison of the different anticoagulants present for the prevention of stroke and systemic embolism in patients with non-valvular atrial fibrillation (FVAC). Results: This tool, which consists of a triptych to be used in Primary Care, wants to be tested within the practices that we perform the next course and thus prove their usefulness.

Key Words: FANV; Anticoagulants; Decision-making; tool; preferences.

\section{Introducción}

Las herramientas de ayuda para la toma de decisiones compartidas son materiales (cuadernillos, folletos, vídeos, DVD, páginas Web) con información fiable (basada en estudios científicos) sobre una determinada enfermedad o problema de salud. Las herramientas sirven para ayudar a las personas a sentirse más informadas y capaces de tomar decisiones sobre su salud, teniendo en cuenta sus valores y preferencias. Además facilitan la relación de las personas afectadas por una enfermedad y los profesionales sanitarios que la asisten, facilitando el proceso de toma de decisiones conjunta (PyDeSalud, 2015).

Las herramientas de ayuda se desarrollan porque muchos problemas de salud cuentan con distintas opciones de tratamiento, cada una con diferentes beneficios y efectos secundarios, que no afectan ni importan a todas las personas de la misma forma. UWHealth, el centro médico académico y el sistema de salud de la Universidad de Winsconsin, es consciente de estos hechos e introduce herramientas de ayuda que proporcionan información clave al paciente (sus opciones, información sobre los medicamentos y cómo pueden ayudarles en su patología, alternativas a la toma de la medicación...) (Healthwise Staff, 2016).

En las enfermedades en las que no existe una única o «mejor» opción de tratamiento, es necesario informar e incorporar las preferencias de las personas afectas para que tomen decisiones junto con los profesionales sanitarios que los asisten (PyDeSalud, 2015).

La herramienta de ayuda sobre anticoagulantes está dirigida a personas con previas patologías cardíacas o riesgo de padecerlas.

La fibrilación auricular (FA) es definida por Medlineplus como una arritmia cardíaca crónica, es decir, un problema con la velocidad o el ritmo de los latidos del corazón. Es debida a una alteración en la contracción de la aurícula, que es una parte del corazón. Esta arritmia es la más frecuente, afecta a un 1-2\% de la población. Su prevalencia se incrementa con la edad, de modo que se estima que la padece un $8,5 \%$ de la población española mayor de 60 años. Los factores de riesgo de la fibrilación auricular son:

- Ser mayor de 60 años de edad.

- Diabetes.

- Problemas cardíacos como: presión arterial alta, enfermedad de las arterias coronarias, ataques al corazón previos, insuficiencia cardíaca congestiva.

- Apnea del sueño.

- Abuso de alcohol o estimulantes. 
Los síntomas de la FA son diferentes para cada persona, muchas no presentan ningún tipo de síntoma. Algunos de ellos son:

- Sentirse demasiado cansado o con falta de energía (el más común).

- Pulso más rápido que el normal o que alterna entre rápido y lento.

- Falta de aire.

- Palpitaciones del corazón (sensación de que el corazón está agitado, late con rapidez o que aletea).

- Problemas al hacer ejercicio o actividades diarias.

- Dolor, presión, opresión o molestia en el pecho.

- Mareos, aturdimiento o desmayos.

La FA confiere a los pacientes que la padecen un riesgo de ictus y embolia sistémica superior en 5 veces al de la población general. Se estima que 1 de cada 5 ictus isquémicos están asociados a la presencia de FA.

El ictus, también llamado accidente cerebrovascular, sucede cuando el flujo de sangre a una parte del cerebro se detiene. Si el flujo sanguíneo se detiene por más de pocos segundos, el cerebro no puede recibir nutrientes y oxígeno. Las células cerebrales pueden morir, lo que causa daño permanente (Tidy, 2017).

La embolia sistémica es una interrupción repentina del flujo de sangre a un órgano o parte del cuerpo debido a un coágulo (émbolo) que viene de otra parte del cuerpo.

Como estas dos patologías pueden provocar daños irreversibles e incluso la muerte del paciente es importante su prevención. Para esta se administran, entre otros fármacos, anticoagulantes.

Los anticoagulantes son fármacos que impiden la coagulación de la sangre, es decir, la formación de coágulos, favoreciendo su disolución con el fin de evitar taponamientos que pueden tener consecuencias graves como ictus, infarto, tromboembolismo pulmonar, entre otras. En general podemos hablar de tres tipos de anticoagulantes: los anticoagulantes orales clásicos (sintrom), los nuevos anticoagulantes orales y las heparinas de bajo peso molecular que se administran por vía subcutánea.

\section{Materiales y métodos}

Con el fin de investigar cuáles son las preferencias de los pacientes en cuanto a lo que ellos considerarían su mejor tratamiento anticoagulante, hemos desarrollado una serie de tablas a modo de cuestionario para que puedan orientar sus decisiones contestando a estas preguntas, y de esta manera hacerlos partícipes de la relación médico-paciente a la hora de decidir dicha terapia. Como se puede observar en la Tabla 1, en primer lugar, nos centramos en los conocimientos que tienen las personas afectadas sobre los diferentes tipos de anticoagulantes: 
Tabla 1

Conocimientos del Paciente: ¿Qué conozco?

Por favor, responda "Sí», «No» o «No lo sé» a las afirmaciones relacionadas con los tratamientos anticoagulantes. No se preocupe si no conoce la respuesta, conteste en base a lo que usted crea correcto, señalando con una $X$ en la casilla que considere oportuna.

Pregunta $\quad$ Sí
1. ¿Conoce la función de los anticoagulantes y para que
patologías se administran?
2. ¿Existen distintas formas u opciones de administrar los
anticoagulantes?
3. ¿Puede causar la tensión arterial alta problemas para
el corazón?
4. ¿Es la hemorragia un efecto secundario de la toma de
anticoagulantes?
5. ¿Debe ser la toma de anticoagulantes igual para todas
las personas independientemente de sus característi-
cas?

Tras la rellenar este cuestionario, se le dará a cada paciente información sobre los diferentes tipos de anticoagulantes:

Anticoagulantes en la Prevención del Ictus en pacientes con FANV:

En primer lugar debemos diferenciar entre 3 tipos de anticoagulantes:

- Anticoagulantes clásicos

- Heparina

- Nuevos anticoagulantes orales (NACOs)

Entre los anticoagulantes clásicos diferenciamos la Warfarina y el Sintrom. Estos presentan como beneficios principales que son los más baratos, que tienen un antídoto específico, que al ser los más antiguos se tiene un mayor conocimiento del fármaco y que existen métodos de control validados. Además se ha comprobado que la warfarina es eficaz para prevenir el accidente cerebrovascular isquémico en pacientes con fibrilación auricular en la práctica clínica diaria. Los resultados de los ensayos aleatorios de anticoagulación se traducen bien en la atención clínica de pacientes con fibrilación auricular (Go y cols., 2003). La vía de administración para ambos es oral, pero varía la posología. Mientras que en la warfarina se pautan $5 \mathrm{mg} /$ día, en el caso del Sintrom debemos variar la dosis según el valor de Quick de cada paciente (más 50\% 2 comprimidos $=8 \mathrm{mg}, 30 \%-40 \% 1$ comprimidos $=4 \mathrm{mg}, 15-30 \% 1 / 4$ o 1 comprimidos $=1-4 \mathrm{mg}$ ).

Entre los inconvenientes que presentan ambos medicamentos destacan:

- Seguimiento de la dieta basado en la ingesta de Vit K. 
- Revisiones médicas periódicas, en el caso del Sintrom para revisar el valor de Quick, y en caso de la Warfarina para el mantenimiento de los niveles del IRN (2-3) (Tidy, 2017).

Ambos presentan ciertos riesgos y efectos secundarios. En el caso del Sintrom destaca el riesgo frecuente de hemorragias y el mal control de la dosis en 1/3 de los pacientes. En el caso de la warfarina existe un mayor riesgo de hemorragias, con mayor incidencia en el cerebro.

En cuanto a la heparina nos centramos en la heparina sódica, que es aquella que presenta una vía de administración intravenosa o subcutánea profunda. La posología de la misma son $250 \mathrm{UI} / \mathrm{Kg} / 12 \mathrm{~h}$. Entre los beneficios de la heparina destaca que no debemos realizar un seguimiento de nuestra dieta ni realizar revisiones médicas periódicas. Además, otro de los beneficios es que es el anticoagulante de elección en embarazadas. En cuanto a los riesgos y efectos secundarios destaca la aparición de hemorragias frecuentes y de edemas si no se rota de zona de pinchazo en la administración de la misma (García, 2013).

Por otro lado, nos encontramos con los nuevos anticoagulantes orales, entre los que podemos destacar el Dabigatrán, Rivaroxabán, Apixabán, Edoxabán. Todos estos nuevos anticoagulantes orales, han surgido teniendo en cuenta que los pacientes con rangos de anticoagulación bajo del nivel terapéutico presentan riesgo de trombosis, mientras que los que tienen niveles superiores al normal, la probabilidad de presentar un sangrado patológico puede llegar a más de $30 \%$ al año. Por todo ello, se están desarrollando nuevos anticoagulantes con farmacocinética y farmacodinamia más predecibles, mayor rango terapéutico y dosis fija que no requieren monitorización. Estos nuevos anticoagulantes se han enfocado en la inhibición específica del factor $X$ ( $F X a)$ y de la trombina (Ila), enzimas claves en la vía final de la coagulación (Berkovits, Aizman, Zúñiga, Pereira y Mezzano, 2011) (Mateo, 2013).

En conjunto, los nuevos anticoagulantes presentan algunas ventajas tales como que tienen un rápido inicio de acción, un efecto anticoagulante predecible, así como pocas interacciones con alimentos y con fármacos. Además, todas ellas presentan una serie de consecuencias clínicas, como que no es necesaria una terapia puente, tampoco es necesaria una monitorización rutinaria, existen bajos riesgos de efectos adversos, tasas de sangrado menores, así como que no implican grandes limitaciones en los fármacos y en los alimentos consumidos. Otra característica positiva a destacar, es que el Dabigatran es el único nuevo anticoagulante sin riesgo para la insuficiencia hepática (García, 2013) (Mateo, 2013).

Por otro lado, otra de las consideraciones importantes de cada uno de ellos, es su posología pudiendo variar su administración oral de 1 a 2 dosis diarias, como por ejemplo:

- Apixabán: 2-5 mg/2 día.

- Edoxabán: $60 \mathrm{mg} / 1$ día.

- Rivaroxabán: 20 mg/1 día.

- Dabigatrán: $75 \mathrm{mg} / 2$ día.

Sin embargo, también estos nuevos anticoagulantes orales presentan unos riesgos y efectos secundarios como podrían ser:

- Menor riesgo de hemorragias frecuentes, pero con mayor incidencia en hemorragias gastrointestinales.

- No existe un método de monopolización validado.

- No existe antídoto para estos nuevos anticoagulantes orales. 
- El Dabigatrán está contraindicado en la insuficiencia hepática (Vidal Vademecum Spain, 2017)

Tabla 2

Comparación de los anticoagulantes clásicos y heparina vs. nuevos

\begin{tabular}{|c|c|c|c|c|c|c|c|}
\hline Anticoagulantes & & Beneficios & Via & Posologia & $\begin{array}{c}\text { Dieta } \\
\text { (Control } \\
\text { Vltamina K) }\end{array}$ & $\begin{array}{l}\text { Revislones } \\
\text { Médicas }\end{array}$ & $\begin{array}{c}\text { Riesgos y efectos } \\
\text { secundarlos }\end{array}$ \\
\hline $\begin{array}{l}\text { Anticoagulantes } \\
\text { Clásicos }\end{array}$ & Simtrom & $\begin{array}{l}\text { Barato y antidoto } \\
\text { especifico } \\
\text { Mayor conocimiento } \\
\text { del fármaco y método } \\
\text { de control validado }\end{array}$ & Oral & $\begin{array}{c}\text { Según el valor de } \\
\text { Quick: } \\
\text { - más 50\% } 2 \text { comp. } \\
8 \mathrm{mg} \\
-30 \%-\% 50 \% 1 \\
\text { comp. }-4 \mathrm{mg} \\
-15-30 \% 1 / 01 \\
\text { comp - } 1-4 \mathrm{mg}\end{array}$ & Si & $\begin{array}{l}\text { Periódicas para } \\
\text { seguimiento del } \\
\text { valor de Quick }\end{array}$ & $\begin{array}{l}\text { Riesgo de hemorragias } \\
\text { frecuentes, } 1 / 3 \text { no está bien } \\
\text { controlado. }\end{array}$ \\
\hline $\begin{array}{l}\text { Anticoagulantes } \\
\text { Clásicos }\end{array}$ & Warfarina & & & $5 \mathrm{mg} / 1 \mathrm{dla}$ & & $\begin{array}{l}\text { Periódicas para } \\
\text { mantenimiento del } \\
\text { IRN }\left(\begin{array}{ll}2.0 & 3.0\end{array}\right)\end{array}$ & $\begin{array}{l}\text { Alto riesgo de hemorragias, } \\
\text { con mayor incidencia en } \\
\text { Corobro }\end{array}$ \\
\hline $\begin{array}{l}\text { Anticoagulantes } \\
\text { Nuevos }\end{array}$ & Aplxaban & $\begin{array}{l}\text { Pocas interacciones } \\
\text { farmacológicas/ } \\
\text { alimentarias } \\
\text { Rápido inicio de acción } \\
\text { No requieren control } \\
\text { de la coagulación } \\
\text { rutinario } \\
\text { Tasas de sangrado } \\
\text { menores } \\
\text { Dabigatrán: Único sin } \\
\text { riesgo para la } \\
\text { insuficiencia hepática }\end{array}$ & & $2-5 \mathrm{mg} / 2 \mathrm{dla}$ & NO & & $\begin{array}{l}\text { Menor riesgo de } \\
\text { hemorragias frecuentes, con } \\
\text { mayor incidencia en } \\
\text { ESTÓMAGO } \\
\text { No existe método de } \\
\text { monopolización validado } \\
\text { No antidoto }\end{array}$ \\
\hline & $\begin{array}{l}\text { Edoxaban } \\
\text { Rlvaroxaban } \\
\text { Dobigatrán }\end{array}$ & & & $\begin{array}{l}60 \mathrm{mg} / 1 \mathrm{dla} \\
20 \mathrm{mg} / 1 \mathrm{dla} \\
75 \mathrm{mg} / 2 \mathrm{dla}\end{array}$ & & & \\
\hline Heparina & $\begin{array}{l}\text { Heparina } \\
\text { sódlca }\end{array}$ & $\begin{array}{c}\text { Uso durante embarazo } \\
\text { y lactancia }\end{array}$ & $\begin{array}{l}\text { Intravenosa/ } \\
\text { subcutánea } \\
\text { profunda en } \\
\text { grasa } \\
\text { abdominal }\end{array}$ & 250 UU/Kg/12 hora & & & $\begin{array}{l}\text { Riesgo de hemorragias } \\
\text { frecuentes, aparición de } \\
\text { edemas si no se rota la } \\
\text { zona de administración }\end{array}$ \\
\hline
\end{tabular}

Con toda esta información los pacientes serán capaces de contestar el siguiente cuestionario, que se centra en saber las preferencias según las características de cada tipo de anticoagulante tabla 3-6.

Atributos como la eficacia, dosis fija y seguridad de los AO son los más valorados por los pacientes con fibrilación auricular no valvular. Estas preferencias deberían considerarse al instaurar o cambiar el tratamiento con $\mathrm{AO}$ para mejorar el cumplimiento y prevención en pacientes (González-Rojas y cols., 2012) 
Tabla 3

Preferencias: cuestionario para el paciente (PyDeSalud, 2015)

A continuación, le presentamos una serie de cuestiones para comprobar sus preferencias sobre los anticoagulantes. Lea atentamente las preguntas y conteste con total sinceridad, valorando cada una del 1 al 10.

1. ¿Qué importancia tiene para usted...

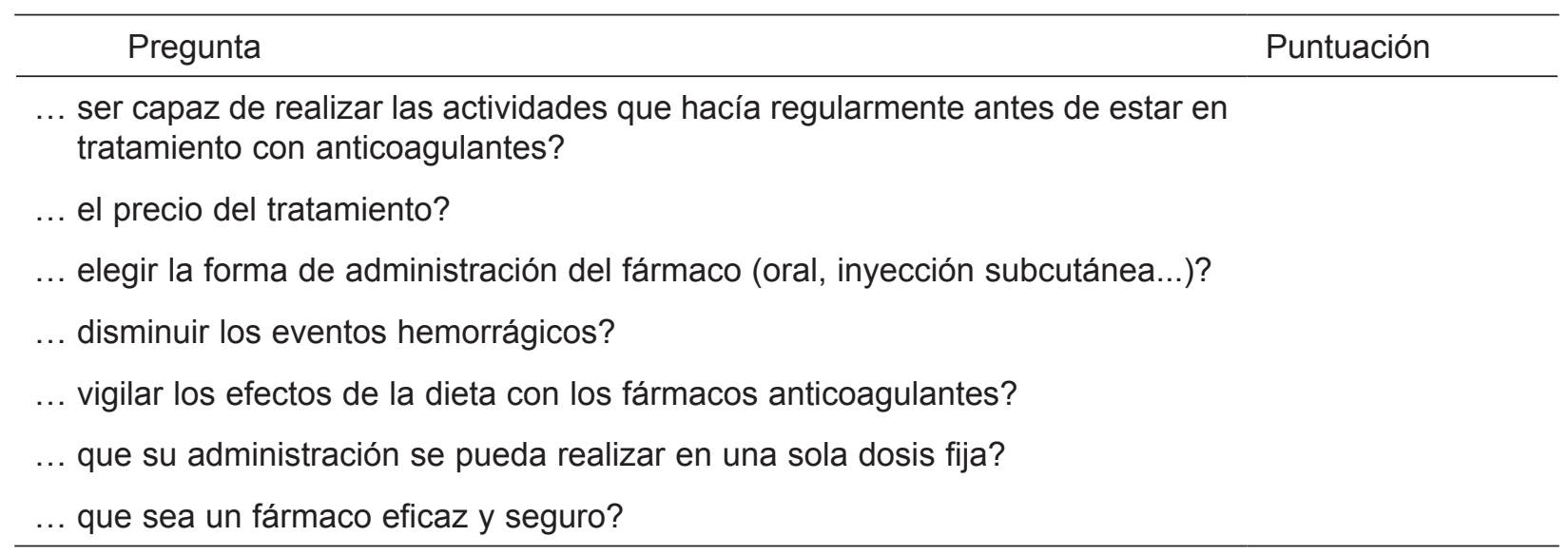

2. ¿Cuánto le preocupa...

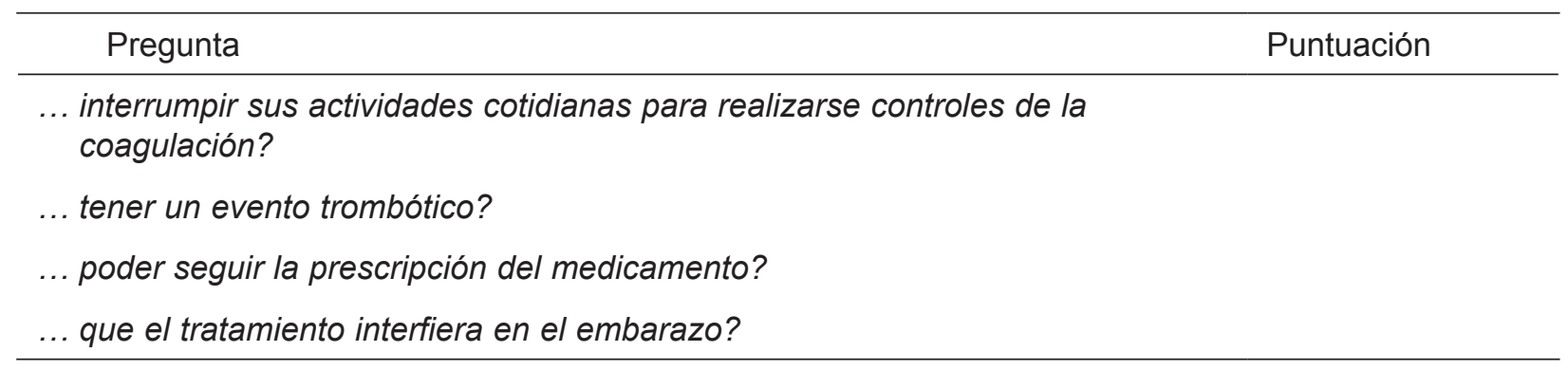

Tabla 4

Decidiendo el tratamiento

Considerando las opciones de tratamiento (SINTROM, heparina, nuevos anticoagulantes) por favor, conteste a las siguientes preguntas marcando la opción de la escala que más se adecúe a su preferencia (PyDeSalud, 2015).

\begin{tabular}{llllll}
\hline Enunciado & $\begin{array}{c}\text { 1.Total } \\
\text { desacuerdo }\end{array}$ & 2 & 3 & 4 & 5.Total acuerdo \\
\hline
\end{tabular}

\footnotetext{
Conozco los tratamientos

disponibles para mí

Conozco los beneficios de

cada opción

Conozco los riesgos y efectos secundarios de cada opción
} 


\begin{tabular}{|c|c|c|c|c|c|c|}
\hline Enunciado & $\begin{array}{c}\text { 1.Total } \\
\text { desacuerdo }\end{array}$ & 2 & 3 & 4 & 5.Total & acuerdo \\
\hline \multicolumn{7}{|l|}{$\begin{array}{l}\text { Tengo claro qué beneficios } \\
\text { son los que más me } \\
\text { importan }\end{array}$} \\
\hline \multicolumn{7}{|l|}{$\begin{array}{l}\text { Tengo claro qué riesgos y } \\
\text { efectos secundarios son los } \\
\text { que más me importan }\end{array}$} \\
\hline $\begin{array}{l}\text { Tengo claro qué es más } \\
\text { importante para mí: los } \\
\text { beneficios o los riesgos/ } \\
\text { efectos secundarios }\end{array}$ & & & & & & \\
\hline
\end{tabular}

Tabla 5

Opciones de tratamiento. Rodee la opción que contempla contestando a la siguiente pregunta (PyDeSalud, 2015)

Si tuviera que elegir un tratamiento ahora, ¿cuál elegiría?

Anticoagulantes Clásicos (Sintrom)

Heparina

Nuevos Anticoagulantes orales

Tabla 6

De acuerdo a sus preferencias, ¿quién prefiere usted que tome la decisión sobre el tratamiento a seguir? (marque con una $X$ sólo una de las siguientes opciones) (PyDeSalud, 2015)

Prefiero ser yo quien tome la decisión sobre el tratamiento que recibiré.

Prefiero ser yo quien tome la decisión sobre mi tratamiento, tras considerar seriamente la opción del profesional sanitario (médico de atención primaria).

Preferiría que el profesional sanitario (médico de atención primaria) y yo compartamos la responsabilidad de elegir cuál es el tratamiento que más me conviene.

Prefiero que el profesional sanitario (médico de atención primaria) tome la decisión final sobre mi tratamiento, pero considerando seriamente mi opinión.

Prefiero dejar todas las decisiones sobre mi tratamiento al profesional sanitario (médico de atención primaria).

Prefiero ser yo quien tome la decisión sobre el tratamiento que recibiré.

\section{Resultados}

Esta herramienta está pensada para utilizarla sobretodo en las consultas de Atención Primaria dado que es aquí dónde más se prescriben estos fármacos. Por tal razón, la idea inicial era probarla en dichas consultas durante nuestro período de prácticas, sin embargo, cuando la tuvimos lista ya las habíamos terminado. 
Hay varios parámetros a explorar en los resultados. El primero sería tener una idea general de los conocimientos de la población acerca de los anticoagulantes antes de que se les haya dado la información. La importancia de ello es saber si los pacientes conocen algo de estos fármacos a priori, y si la mayoría no los conocen, plantearse la educación social mediante la realización de charlas y/o talleres en el centro de salud.

El segundo parámetro se centraría en investigar si los pacientes son capaces de, con la información facilitada, discernir cuál sería el mejor tratamiento para ellos valorando cómo puede afectar el fármaco a cada aspecto de su vida, y de esta manera, llegar a elegir por sí mismos el anticoagulante idóneo para ellos. Si fuera así, se demostrarían dos cosas: el gran entendimiento que se puede alcanzar sobre cada fármaco que llevaría a una mayor adherencia por parte de los pacientes, facilitando así la faena del médico; y la gran independencia que pueden llegar a tener los pacientes si se les explican correctamente las cosas de manera que puedan entenderlas.

En tercer lugar y en referencia al último punto del cuestionario, nos planteamos cuán importante es para los usuarios la relación con su médico de Atención Primaria y el grado de satisfacción en esta. Así, dependiendo de quién prefieran que elija el tratamiento, veremos distintos tipos de relación. Por ejemplo, si el paciente quiere elegir por sí mismo podemos imaginar que se trata de una relación en la que el paciente no está muy satisfecho y hay poca confianza, por ello prefiere no contar con el médico; si decide que sea una responsabilidad compartida entre los dos, pensaremos que estamos ante una buena relación médico-paciente, ya que son capaces de dialogar y decidir entre los dos qué es lo mejor; y si el paciente prefiere que decida el médico por él, tal vez estemos ante una relación con demasiada confianza depositada en el profesional sanitario y seguramente con un alto grado de satisfacción por parte del usuario, sin embargo no se trataría de la relación horizontal que se busca actualmente con nuestros pacientes.

\section{Referencias bibliográficas}

Berkovits, A., Aizman, A., Zúñiga, P., Pereira, J. y Mezzano, D. (2011). Nuevos anticoagulantes orales. Revista médica de Chile, 139(10), 1347-1355.

Dabigatran etexilato (2017). Vidal Vademecum Spain. Recuperado de: www.vademecum.es// principios-activos-dabigatran+etexilato-b01ae07.

Fibrilación auricular. Recuperado de: www.medlineplus.gov/spanish/atrialfibrillation.

García, J. P. (2013). Nuevos anticoagulantes frente a anticoagulantes clásicos: ventajas e inconvenientes. SEMERGEN-Medicina de Familia, 39(1), 10-16.

Go, A.S., Hylek, E.M., Chang, Y., Phillips, K.A., Henault, L.E., Capra, A.M., Jensvold, N.G., Selby, J.V. y Singer, D.E. (2003). Anticoagulation Therapy for Stroke Prevention in Atrial Fibrillation: How Well Do Randomized Trials Translate Into Clinical Practice?. JAMA, 290(20), 2685-2692.

González-Rojas, N., Giménez, E., Fernández, M.A., Heineger, A.I., Martínez, J.L., Villar, J. y Lizán, L. (2012). Preferencias por los tratamientos anticoagulantes orales para la prevención a medio y largo plazo del ictus en la fibrilación auricular no valvular. Revista de Neurología, 55 (1), 11-19.

Healthwise Staff. Atrial Fibrillation: Should I Take an Anticoagulant to Prevent Stroke? (2016). UW Health. Recuperado de: www.uwhealth.org/health/topic/decisionpoint/atrial-fibrillation-should-i-take-an-anticoagulant-to-prevent-stroke/tx2209.html.

Mateo, J. (2013). Nuevos anticoagulantes orales y su papel en la práctica clínica. Revista Española de Cardiología, 13(C), 33-41. 
Tidy, C. Preventing Stroke When You Have Atrial Fibrillation. (2017). Patient.info. Recuperado de: www.patient.info/health/preventing-stroke-when-you-have-atrial-fibrillation.

Toma de decisiones en depresión (2015). PyDeSalud. Recuperado de www.pydesalud.com/ toma-de-decisiones-compartida-en-depresion. 